\title{
RESISTENCIA Y ELASTICIDAD A LA FLEXIÓN DE LA GUADUA ANGUSTIFOLIA KUNTH DE PITALITO, HUILA
}

\author{
BENDING STRESS AND ELASTICITY OF THE GUADUA ANGUSTIFOLIA KUNTH \\ OF PITALITO, HUILA
}

Eduard Sapuyes*

Jesús Osorio**

Caori Takeuchi ${ }^{\text {*wa }}$

Mauricio Duarte

Wilson Erazo

Recibido: 13 de diciembre de 2017

Aceptado: 23 de abril de 2018

\section{Resumen}

Se evaluó resistencia última y módulo de elasticidad a la flexión en muestras de Guadua angustifolia Kunth procedentes del municipio de Pitalito (Huila). Desde la obtención de las muestras hasta los ensayos, se procedió según las normas técnicas colombianas NTC 5300, Cosecha y Postcosecha del Culmo de Guadua angustifolia Kunth, y NTC 5525, Métodos de Ensayo para Determinar las Propiedades Físicas y Mecánicas de la Guadua angustifolia Kunth, y el titulo G, "Estructuras de madera y estructuras de guadua”, del Reglamento Colombiano de Construcciones Sismorresistentes. Se encontraron valores de esfuerzo admisible y módulo de elasticidad promedio de $13.85 \mathrm{MPa}$ y $15483 \mathrm{MPa}$, respectivamente, para contenidos de humedad promedio de $102 \%$. Adicionalmente se realizaron comparaciones con otras investigaciones realizadas en Colombia.

Palabras claves: Guadua angustifolia, culmo, esfuerzo último, esfuerzo de flexión, módulo de elasticidad.

\begin{abstract}
Ultimate strength and flexural modulus of elasticity were evaluated in samples of Guadua angustifolia Kunth from the municipality of Pitalito, Huila. From obtaining samples to testing, the recommendations of the Colombian Technical Standard NTC 5300, Harvest and post-harvest of the culm of Guadua angustifolia Kunth, NTC 5525, Test methods for the determination of physical and mechanical properties of the Guadua angustifolia Kunth and the NSR 10 "Reglamento
\end{abstract}

\footnotetext{
* Ingeniero agrícola. Universidad Surcolombiana, Neiva, Colombia. https://orcid.org/0000-0002-2894-843X. eduard1229@outlook.com

** Ingeniero Agrícola. Universidad Surcolombiana, Neiva, Colombia. https://orcid.org/0000-0002-2494-7659. jesda1194@ hotmail.com

*** Ph. D. en Ingeniería, Ciencia y Tecnología de Materiales. Docente Universidad Nacional de Colombia, Bogotá, Colombia. https://orcid.org/0000-0002-6273-7118. cptakeuchit@unal.edu.co

${ }^{* \star * \star}$ Magíster en Ingeniería Civil. Docente Universidad Surcolombiana, Neiva, Colombia. https://orcid.org/00000003-1801-6513.maduto@usco.edu.co

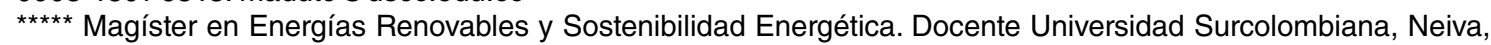
Colombia. https://orcid.org/0000-0001-7374-974X. wilson.erazo@usco.edu.co
} 
Colombiano de Construcciones Sismo Resistente" Chapter G "Estructuras de madera y estructuras de guadua" were followed. The values of the allowable stress and the modulus of elasticity of $13.85 \mathrm{MPa}$ and $15483 \mathrm{MPa}$, respectively, were found, for average moisture content of $102 \%$. Additionally, comparisons with other investigations carried out in Colombia were made.

Key words: Guadua angustifolia, stem, ultimate strength, bending stress, modulus of elasticity.

\section{INTRODUCCIÓN}

El interés en la Guadua angustifolia Kunth surgió de la buena respuesta estructural de las edificaciones construidas con elementos de este material a los sismos que han afectado al Eje Cafetero colombiano. Una muestra de esta capacidad son las instalaciones que no han colapsado a pesar de las múltiples actividades sísmicas (Reyes y Rayo, 2014)

Se trata de un material vegetal que se destaca por su flexibilidad, resistencia, economía y fácil obtención en algunas regiones del mundo, cuya utilización en construcciones es habitual. $\mathrm{Al}$ respecto, existen estándares nacionales e internacionales que presentan procedimientos para la evaluación de su resistencia y deformación, factores que se deben revisar dada la variabilidad de las condiciones naturales en que se sustenta el material (Chavarro, 2016).

Según Vélez (2001), la Guadua angustifolia puede usarse en el área de construcción, es un recurso inagotable y estructural, apropiado para vivienda y otros tipos de edificaciones. Superior al concreto en compresión, la guadua admite grandes deflexiones, por lo que es un material ideal para realizar construcciones sismoresistentes, seguras y económicas.

Aunque las propiedades mecánicas de la guadua dependen de la especie botánica a la que pertenece, la resistencia a flexión también depende de la edad de corte de la planta, la sección del culmo que se utilice y de las propiedades físicas como son la humedad, la densidad básica y el peso específico. (Prieto y Sánchez, 2002, p. 13)

Se ha comprobado la importancia de la guadua como alternativa para resolver las necesidades de vivienda. Sus propiedades antisísmicas han despertado interés y han captado la atención de investigadores. Igualmente, se han establecido normas y procedimientos al respecto de su uso. Es un recurso importante de la industria de los laminados, incluso se comprobó que los recursos energéticos necesarios para la construcción de una vivienda son menores con guadua que con concreto (Villegas, 2005).

En la actualidad la explotación de la guadua en Colombia ha venido mostrando avances importantes, se puede mencionar que dicho potencial se empezó a mostrar a través de los diferentes proyectos de construcción posteriores al terremoto del Eje Cafetero aprovechando su cualidad sismo resistente; y en investigaciones sobre su aporte a la conservación del medio ambiente y sobre sus fortalezas físicomecánicas para usos industriales. Al mismo tiempo demuestra que es un producto que representa una alternativa de diversificación económica viable para los agricultores colombianos. (Brand, Ruiz y Lozano, 2015, p. 10)

Según McClure (1966), una de las especies de bambú más usadas para la construcción es el bambú guadua. Esta especie se encuentra en países asiáticos como China,Japón o Corea, así como en países latinoamericanos como Costa Rica, Panamá o Nicaragua. Este tipo de bambú guadua se 
presenta en tres variedades: Dendrocalamus, Guadua angustifolia y Guadua aculatada o amplexifolia, las cuales presentan características muy similares.

Los estándares para la determinación de las propiedades físicas y mecánicas del bambú y la guadua son: ISO 22157. Bamboo: Determination of Physical and Mechanical Properties on Bamboo (ISO, 2004), Métodos de Ensayo para Determinar las Propiedades Físicas y Mecánicas de la Guadua angustifolia Kunth. NTC 5525 (ICONTEC, 2008) y el capítulo G.12 del Reglamento Colombiano de Construcción Sismo Resistente NSR-10 (Ministerio de Ambiente, Vivienda y Desarrollo Territorial, 2010).

En el proyecto tomado de referencia para este estudio, "Esfuerzo de flexión y módulo de elasticidad de la Guadua angustifolia Kunth procedentes del municipio de Pitalito, Huila", se implementaron los procedimientos establecidos por las normas técnicas colombianas NTC 5300, Cosecha y Postcosecha del Culmo de Guadua angustifolia Kunth y NTC 5525, Métodos de Ensayo para Determinar las Propiedades Físicas y Mecánicas de la Guadua angustifolia Kunth, y el título G, "Estructuras de madera y estructuras de guadua", del Reglamento Colombiano de Construcciones Sismoresistente.

En esta zona cafetera del sur del departamento del Huila se encuentran los municipios de Pitalito y Timaná, los cuales fueron seleccionados para el desarrollo de la investigación "Esfuerzo de flexión y módulo de elasticidad en muestras de Guadua angustifolia Kunth procedentes del municipio de Pitalito, Huila", proyecto que da continuidad a las investigaciones realizadas por Capera y Erazo (2012), Duarte, Erazo y Takeuchi (2013), Alarcón y Olarte (2013), López y Salcedo, (2016), entre otros.

En esta publicación se presentan los resultados de ensayos de flexión en la Guadua angustifolia Kunth, realizados en la sede Bogotá de la Universidad Nacional de Colombia.

\section{Metodología}

Se tomaron muestras de material vegetal en la zona rural de los municipios de Pitalito y Timaná del departamento del Huila, ubicados a una altura entre 1000-1800 m s. n. m. y con una temperatura media anual de $18-21^{\circ} \mathrm{C}$.

\section{Localización zonas de estudio}

En total se seleccionaron cuatro rodales (zona de estudio A, B, C y D), tres de ellos localizados en el municipio de Pitalito y uno en el municipio de Timaná (tabla 1 ).

Tabla 1. Localización zonas de estudio

\begin{tabular}{lllcc}
\hline \multirow{2}{*}{ Predio } & Municipio & \multicolumn{2}{c}{ Coordenadas Magna-Sirgas } & Altitud \\
\cline { 2 - 3 } & & Latitud (N) & Longitud (O) & (m s. n. m) \\
\hline A: La Dalia & Pitalito & $1^{\circ} 47^{\prime} 13.80^{\prime \prime}$ & $76^{\circ} 04^{\prime} 03.40^{\prime \prime}$ & 1294 \\
B: Villa María & Pitalito & $1^{\circ} 50^{\prime} 26.11^{\prime \prime}$ & $76^{\circ} 01^{\prime} 58.31^{\prime \prime}$ & 1279 \\
C: La Vega & Timaná & $1^{\circ} 54^{\prime} 19.80^{\prime \prime}$ & $75^{\circ} 58^{\prime} 02.80^{\prime \prime}$ & 1253 \\
D: Sena Yamboró & Pitalito & $1^{\circ} 53^{\prime} 36.00^{\prime \prime}$ & $76^{\circ} 05^{\prime} 25.10^{\prime \prime}$ & 1331 \\
\hline
\end{tabular}

Fuente: Capera y Erazo (2012). 


\section{Normas y protocolos}

Para la selección, corte y aprovechamiento forestal, así como para la caracterización física y mecánica de la Guadua angustifolia, se consultó la reglamentación y estándares vigentes que se muestran en la tabla 2.

Tabla 2. Descripción de las normas utilizadas en el proyecto

\begin{tabular}{ll}
\hline \multicolumn{1}{c}{ Denominación } & \multicolumn{1}{c}{ Título } \\
\hline ISO/TC 165N315 & $\begin{array}{l}\text { Laboratory Manual on Testing Methods for Determination of Physical and Mechanical } \\
\text { Properties of Bamboo } \\
\text { NTC5300 }\end{array}$ \\
NTC5301 & $\begin{array}{l}\text { Cosecha y Postcosecha del Culmo de Guadua angustifolia Kunth } \\
\text { NTC5525 }\end{array}$ \\
NSR10 & $\begin{array}{l}\text { Métodos de Ensayo para Determinar las Propiedades Físicas y Mecánicas de la Guadua } \\
\text { angustifolia Kunth }\end{array}$ \\
\hline
\end{tabular}

Igualmente se consultó la Norma Unificada para el Manejo y Aprovechamiento de la Guadua (Ministerio de Ambiente, Vivienda y Desarrollo Territorial, CVC, CORPOCALDAS, CARDER y CORTOLIMA, 2008) que reúne cinco resoluciones expedida por cinco corporaciones autónomas regionales: Resolución n. ${ }^{\circ}$ 185/2008 (CORPOCALDAS), Resolución n.o 666/2008 (CRQ), Resolución n. ${ }^{\circ}$ 944/2008 (CARDER), Resolución n. ${ }^{\circ}$ 1150/2008 (CORTOLIMA) y CVC Resolución n. ${ }^{\circ}$ 01000439/2008 (CVC).

\section{Selección y corte de culmos}

En los rodales objeto de estudio, se seleccionaron e identificaron las guaduas maduras o hechas (entre 3 y 6 años de edad) que no presentaran daños físicos. Se marcaron con un anillo de pintura naranja a $1 \mathrm{~m}$ de altura a partir del cuello de la guadua (ver figura 1).

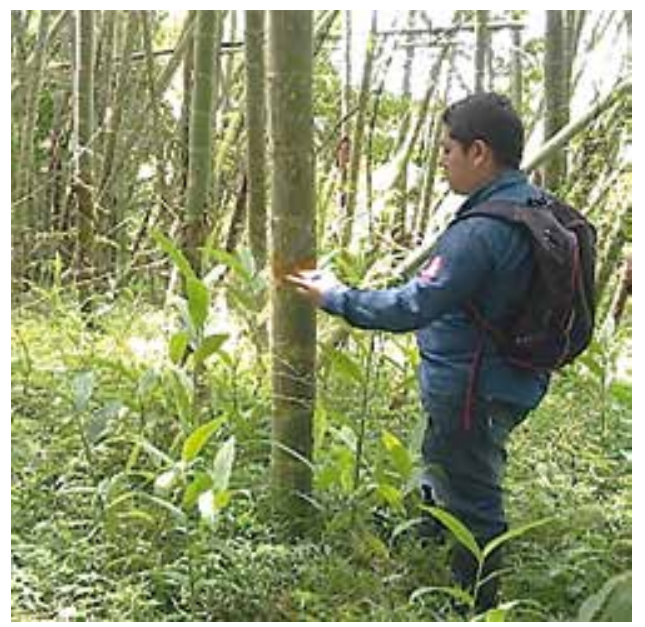

Figura 1. Selección de la guadua (predio La Vega, Timaná, Huila).

El corte de los culmos se realizó con machete a una altura a $1 \mathrm{~m}$ por encima del suelo. Posterior al corte, los culmos se sometieron al proceso de avinagrado in situ durante 2 semanas. Al final, se 
midieron los tallos, se marcaron las 3 secciones longitudinales: cepa $(1.5 \mathrm{~m})$, basa $(4.5 \mathrm{~m})$ y sobrebasa $(3.0 \mathrm{~m})$, y se realizó la caracterización.

\section{Obtención de las probetas}

El material fue transportado desde la zona de estudio hasta las instalaciones de la Universidad Nacional de Colombia, sede Bogotá, edificio SINDU. La preparación de las probetas para el ensayo a flexión se realizó según la norma NTC-5525. Los ensayos se realizaron en la máquina universal Hung Ta Instrument Co. Ltd. modelo 2010

\section{Descripción del ensayo}

Se ensayaron 75 probetas a carga máxima para determinar la resistencia última a flexión y el módulo de elasticidad. Para los ensayos se utilizaron probetas de la sección media de los culmos. La carga se aplicó lo más cerca posible a los tercios medios de cada probeta, a una velocidad constante de $0.5 \mathrm{~mm} / \mathrm{s}$, conforme a lo señalado por la norma NTC 5525. Después de ensayar cada probeta, se seleccionaron muestras de las mismas para evaluar su contenido de humedad y densidad. La disposición de las probetas se realizó tal como se ilustra en la figura 2.

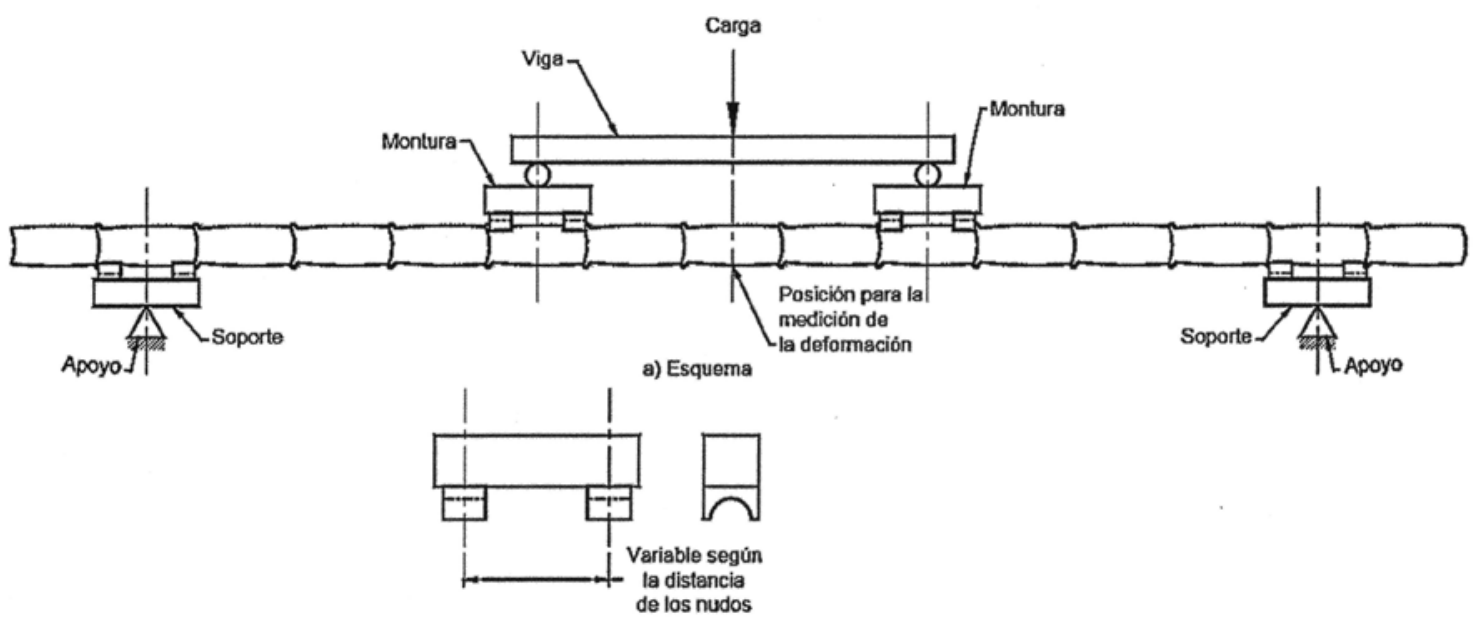

Figura 2. Esquema del ensayo de flexión NTC 5525.

De acuerdo con la NTC 5525, el ensayo debía ser de flexión en cuatro puntos y en los soportes se debía permitir que el culmo rotara libremente, razón por la cual la configuración de los soportes quedó tal como se presenta en la figura 3. 


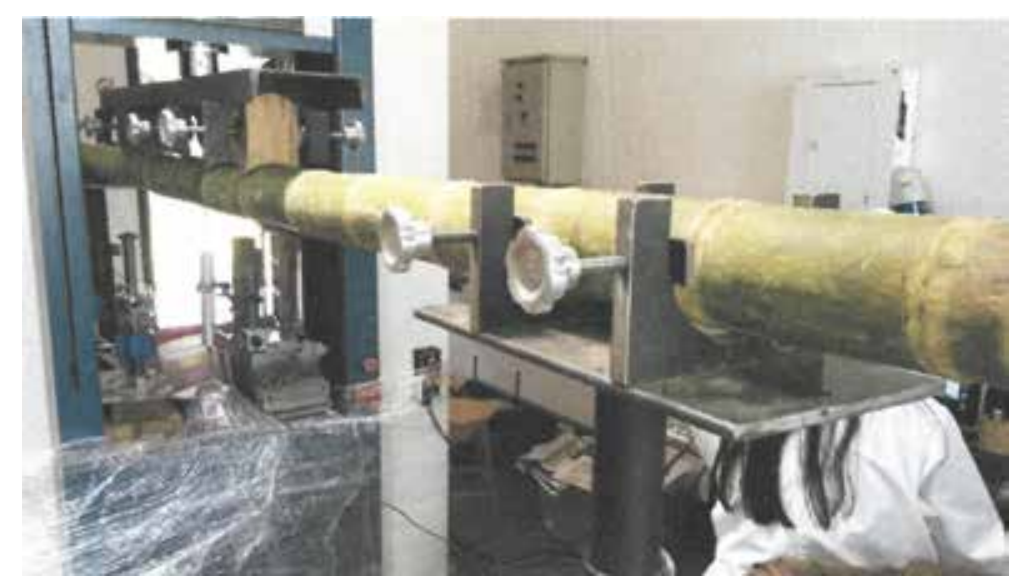

Figura 3. Probeta de guadua apoyada en sus extremos y con aplicación de la carga en el tercio medio.

Los ensayos se realizaron hasta la carga última con el fin de analizar el comportamiento elástico del material. Las gráficas esfuerzo vs. deformación, elaboradas por el ordenador adaptado a la máquina universal, permitieron realizar una primera aproximación (figura 4).

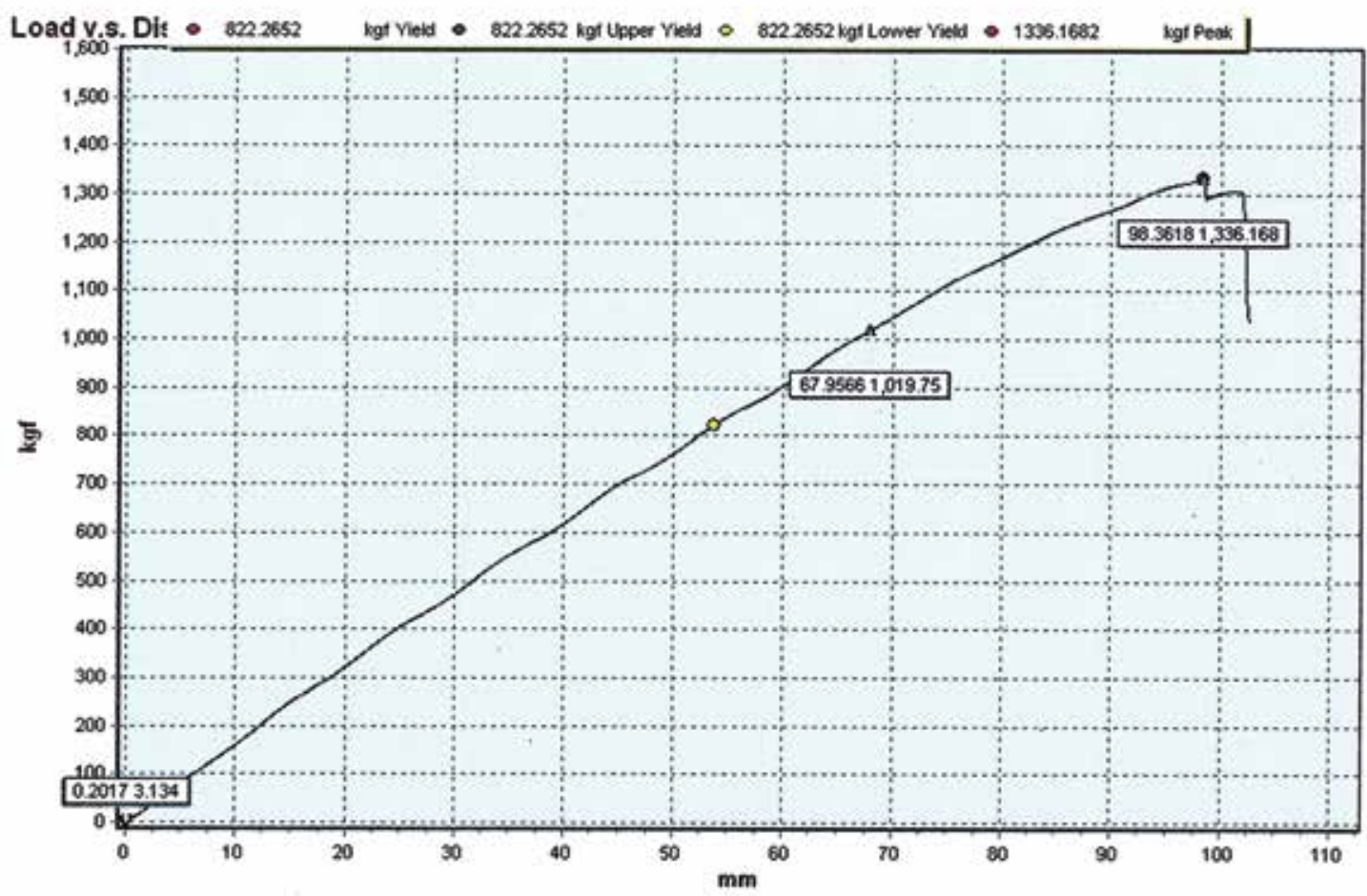

Figura 4. Gráfico esfuerzo vs. deformación.

\section{Esfuerzo normal a flexión y módulo de elasticidad}

Para la determinación del esfuerzo a flexión y módulo de elasticidad de las probetas ensayadas, se tomó como referencia las investigaciones de Prieto y Sánchez (2002) "Comportamiento de la 
Guadua angustifolia sometida a flexión", y Rusinque (2007) "Influencia de la perforación, para inmunización en los tabiques, en el comportamiento de elementos solicitados a flexión de Guadua angustifolia". Para los cálculos se emplearon las ecuaciones 1 y 2.

Ecuación 1. Esfuerzo normal a flexión

$$
\sigma=\frac{32 M_{\max } D_{e}}{\pi\left(D_{E}^{4}-\left(D_{e}-2 t\right)^{4}\right)}
$$

Donde:

$\sigma \quad$ : esfuerzo normal, $\mathrm{Pa}$

$\mathrm{M}_{\max }$ : momento flexionante, $\mathrm{N}-\mathrm{m}$

$\mathrm{D}_{\mathrm{e}} \quad$ : diámetro externo, $\mathrm{m}$

$\mathrm{T}$ : espesor de la pared, $\mathrm{m}$

Ecuación 2. Módulo de elasticidad

$$
E=m\left(\frac{\alpha}{L I}+\frac{12 \beta}{L K A}\right)
$$

Donde: la expresión en paréntesis depende de la disposición de las cargas aplicadas y de la geometría de la sección transversal en el punto donde se tomaron lecturas de desplazamiento, es decir, el centro de la luz y la longitud de la probeta.

Igualmente:

$\mathrm{m}$ : pendiente de la recta carga vs. desplazamiento, dentro del rango elástico

\section{Valor característico y percentil 5}

Los datos de resistencia última a flexión y contenidos de humedad se analizaron de manera exploratoria para excluir valores atípicos; igualmente, se determinaron valores promedios mínimos y máximos y medidas de dispersión mediante análisis descriptivo, con el fin de aplicarlos en las ecuaciones 3 y 4 para el cálculo de valores característicos y percentil 5, respectivamente.

Ecuación 3. Valor característico ${ }^{1}$

$$
f_{k b}=f_{0,05 b}\left[1-\frac{2.7 \frac{s}{m}}{\sqrt{n}}\right]
$$

Donde:

$\mathrm{f}_{0.05 \mathrm{i}} \quad$ : valor correspondiente al percentil 5 de los datos $(\mathrm{MPa})$

$\mathrm{m}$ : promedio

\footnotetext{
${ }^{1}$ Ecuación derivada de Reglamento Colombiano de Construcciones Sismo Resistente. NSR 10 (Ministerio de Ambiente, Vivienda y Desarrollo Territorial, 2010).
} 
s : desviación estándar

$\mathrm{n} \quad$ : número de ensayos

Ecuación 4. Percentil $5^{2}$

$$
f_{0.05 b}=F_{c} *(1-(1.645 * C O V c))
$$

Donde:

Fc : resistencia última a flexión promedio $(\mathrm{MPa})$

$\mathrm{COV}$ : coeficiente de variación de la resistencia última a flexión

\section{Análisis estadístico (análisis de varianza simple)}

Para el análisis estadístico se utilizó un diseño de varianza simple totalmente aleatorio, en el que se evaluaron los valores de resistencia última a flexión de las probetas ensayadas. Previamente se descartaron mediante análisis exploratorio los valores atípicos. Las variables para el diseño ANOVA simple, los factores y niveles de factor se muestran en la tabla 3.

Tabla 3 Factores, niveles de factor y variable dependiente

\begin{tabular}{rlr}
\hline \multicolumn{1}{r}{ Factor } & \multicolumn{1}{c}{ Niveles de factor } & \multicolumn{1}{c}{ Variable dependiente } \\
\hline \multirow{3}{*}{ Zona } & La Dalia & \\
& Villa María & Resistencia última a flexión (MPa) \\
& La Vega & \\
& SENA- Yamboro & \\
\hline
\end{tabular}

El nivel de confiabilidad mínimo para los resultados es del $95 \%$. Los datos se organizaron y procesaron en el software Statgraphics Centurion XVI (versión evaluación), asumiendo como hipótesis la normalidad, homocedasticidad, Levene e independencia de los mismos.

\section{Resultados}

Se presentan a continuación los resultados del análisis estadístico descriptivo realizado para la resistencia última a flexión y el módulo de elasticidad, así como los resultados de otros estudios en relación al módulo de elasticidad para efectos comparativos.

Se observó que las probetas ensayadas a flexión presentaron una densidad básica promedio de $1.10 \mathrm{~kg} / \mathrm{cm}^{3}$ con una desviación estándar de $0.036 \mathrm{~kg} / \mathrm{cm}^{3}$ y contenidos de humedad promedio de $102.2 \%$ con una desviación estándar de $18.03 \%$. Se descartaron cinco probetas (A17, B2, C11, D4 y D12) mediante análisis exploratorio por presentar valores atípicos; por tal motivo, se analizaron 70 probetas en el ANOVA simple. La aleatoriedad en el muestreo, la obtención de probetas y la ejecución de ensayos garantizaron la normalidad, la independencia y la heterocedasticidad.

\section{ANOVA simple}

En el análisis de varianza simple presentado en la tabla 4 se identificó una diferencia estadísticamente significativa entre la media de resistencia última a flexión entre un nivel de procedencia y otro, con un nivel del $95 \%$ de confianza, debido a que el valor-P de la prueba fue menor que 0.05 .

\footnotetext{
${ }^{2}$ Ecuación derivada de Reglamento Colombiano de Construcciones Sismo Resistente. NSR 10 (Ministerio de Ambiente, Vivienda y Desarrollo Territorial, 2010).
} 
Tabla 4. Análisis de varianza simple para esfuerzo a flexión

\begin{tabular}{lccccc}
\hline \multicolumn{1}{c}{ Fuente } & Suma de cuadrados & GI & Cuadrado medio & Razón-F & Valor-P \\
\hline Entre grupos & 1355.16 & 3 & 451.719 & 7.43 & 0.0002 \\
Intra grupos & 4011.78 & 66 & 60.7846 & & \\
\hline Total (corregido) & 5366.94 & 69 & & & \\
\hline
\end{tabular}

\section{Análisis de la resistencia última a flexión y módulo de elasticidad}

En la tabla 5 se muestra la información necesaria para llegar al cálculo de los valores característicos, resistencia última a flexión promedio, desviación estándar, coeficiente de variación, esfuerzos admisibles y número de ensayos, junto con el percentil 5 y los valores característicos. En la tabla 6 se evidencian los resultados del análisis descriptivo para el módulo de elasticidad.

Tabla 5. Análisis resistencia última a flexión

\begin{tabular}{lccccc}
\hline \multicolumn{1}{c}{ Procedencia } & La Dalia & Villa María & La Vega & SENA & Total \\
\hline Casos (n) & 19 & 19 & 19 & 13 & 70 \\
Resistencia última promedio (MPa) & 58.34 & 56.28 & 54.04 & 66.78 & 58.18 \\
Desviación estándar (s) & 7.41 & 8.52 & 8.17 & 6.53 & 8.82 \\
Coeficiente de variación (\%) & 12.71 & 15.14 & 15.12 & 9.78 & 15.16 \\
Percentil 5 (MPa) & 46.15 & 42.27 & 40.61 & 56.04 & 43.68 \\
Valor característico (MPa) & 42.51 & 38.30 & 36.80 & 51.93 & 41.54 \\
Esfuerzo admisible (Ma) & 14.17 & 12.77 & 12.27 & 17.31 & 13.85 \\
\hline
\end{tabular}

Tabla 6. Análisis descriptivo módulo de elasticidad

\begin{tabular}{|l} 
Eecuento \\
Desviación estándar $(\mathrm{MPa})$ \\
Coeficiente de variación \\
Mínimo $(\mathrm{MPa})$ \\
Máximo $(\mathrm{MPa})$ \\
Rango \\
E $0.05(\mathrm{MPa})$
\end{tabular}

70
15480
1750
$11.28 \%$
10530
18710
8190
12560

\section{Comprobación de hipótesis}

\section{Normalidad de los residuos}

La distribución normal de los datos se verifica mediante el gráfico de probabilidad normal de los residuos (figura 5), en el cual se observa los datos acomodados a lo largo de la línea de referencia que pasa a través de la mediana (determinada por el rango intercuartilico). 
Gráfico de probabilidad normal

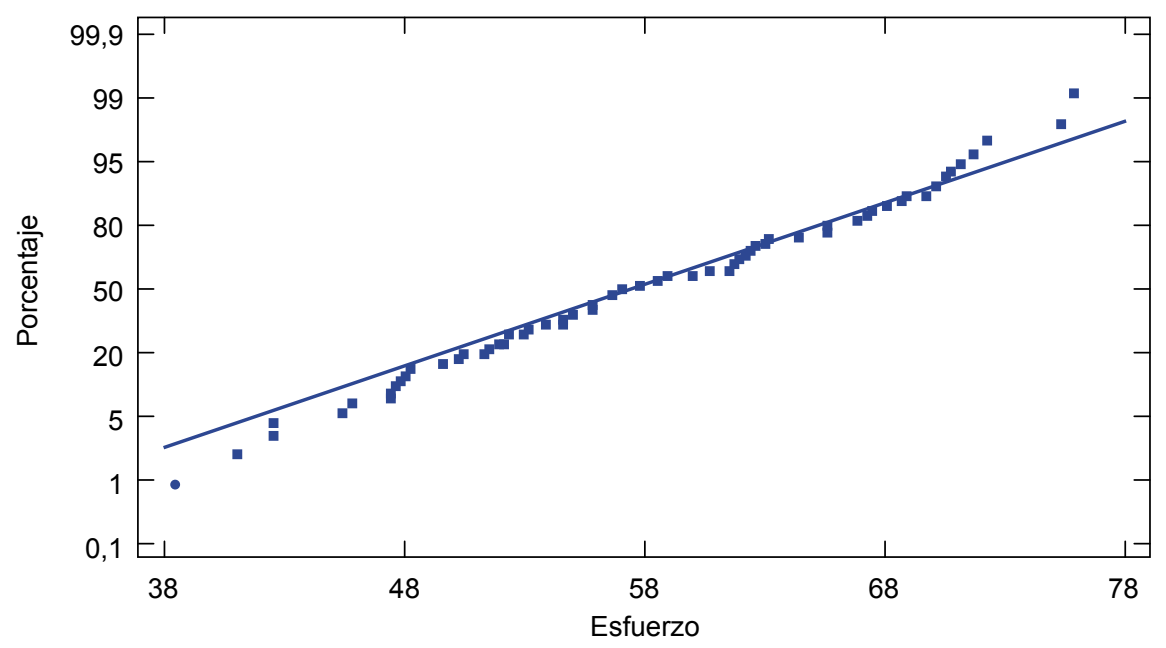

Figura 5. Probabilidad normal para residuos.

\section{Prueba de homocedasticidad}

La homocedasticidad se verificó mediante el gráfico de residuos frente a valores predichos de resistencia (figura 6). Se observa que no existe un patrón general en forma de embudo formado por los puntos; esto demuestra que no hay indicios de heterocedasticidad, es decir, las varianzas de los tres grupos resultan homogéneas.

Gráfico de residuos para esfuerzo

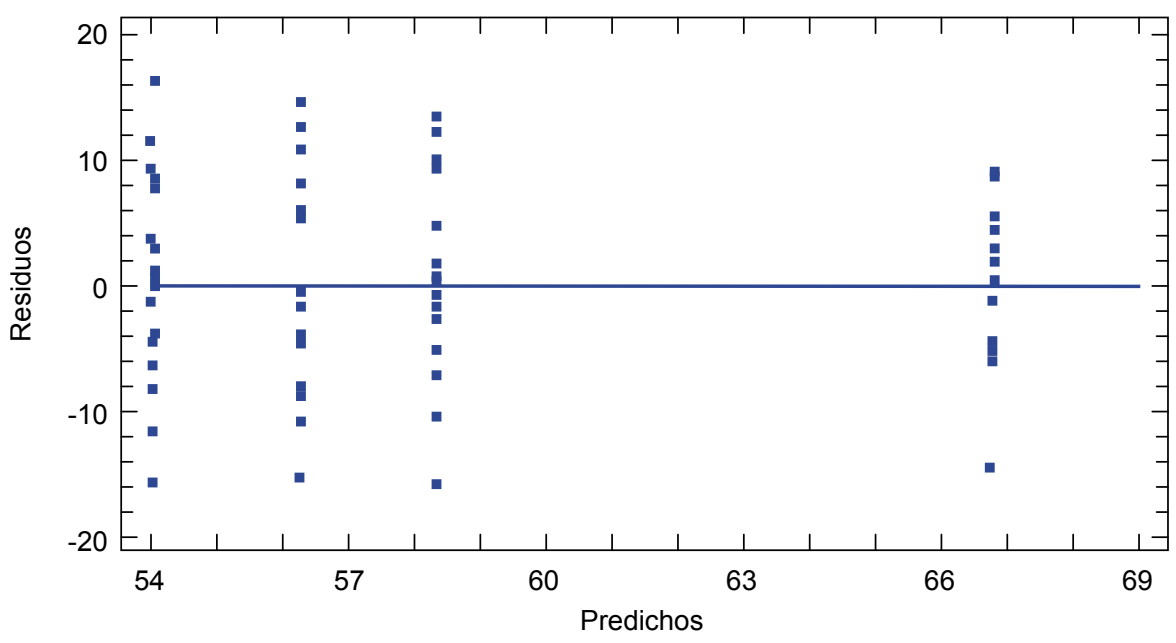

Figura 6. Residuos frente a predichos para esfuerza a flexión en MPa.

Al igual que González, Montoya y Bedoya (2008), Córdoba (2009), Capera y Erazo (2012) y Rojas (2013), la homogeneidad de las varianzas se verificó con la prueba de Levene, cuyo resultado fue $\mathrm{p}=0.485$, lo que implica que no existe ningún indicio significativo de heterocedasticidad. 


\section{Independencia de los residuos}

La aleatoriedad durante la selección de las zonas, culmos y lugares de corte para la extracción de las probetas se evidencia en la dispersión de los puntos en el gráfico residuos frente número de fila (figura 7). Con esto se demuestra la representatividad de las muestras; además, se confirma que no existe ninguna correlación serial entre los datos, es decir, no existe dependencia entre las observaciones.

Gráfico de residuos para esfuerzo

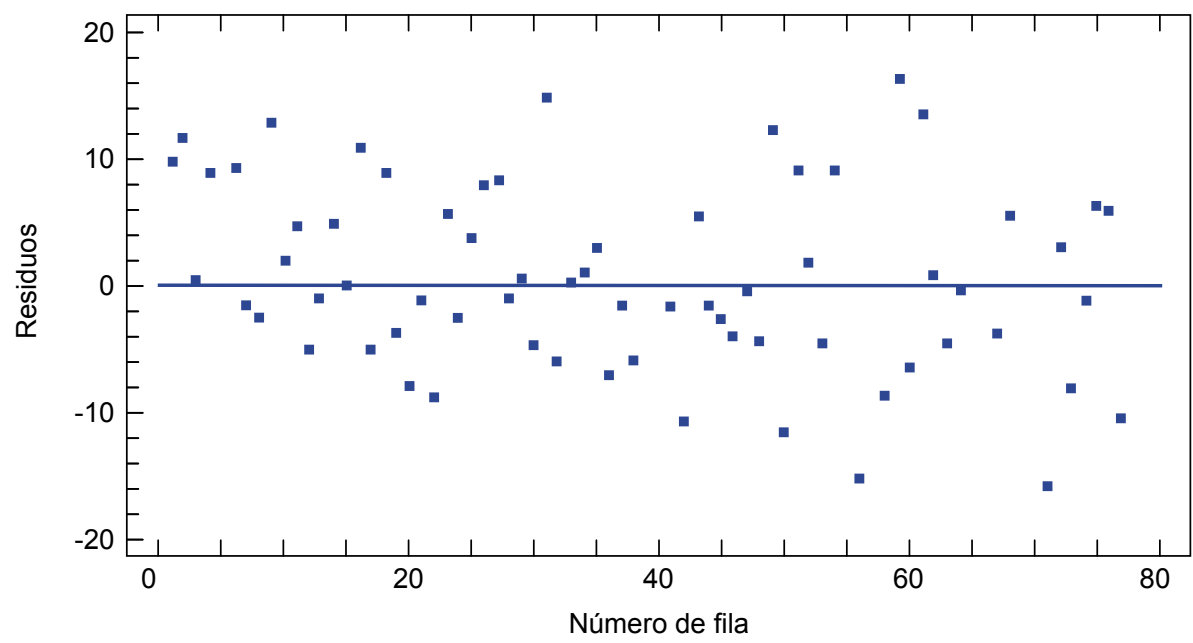

Figura 7. Residuos frente a número de fila para esfuerzo a flexión en MPa.

\section{Módulo de elasticidad en otras investigaciones}

Se presentan los valores de módulo de elasticidad en probetas de Guadua angustifolia con relación diámetro/longitud 1:30 en estudios ajustados a las metodologías de la NTC 5525 (ver tabla 7). Las probetas analizadas tienen una carga aplicada en los tercios medios y pueden girar libremente sus extremos, pero no pueden moverse transversalmente al eje; igualmente, un extremo de la viga puede moverse en dirección axial o longitudinal, como se ilustra en la figura 8.

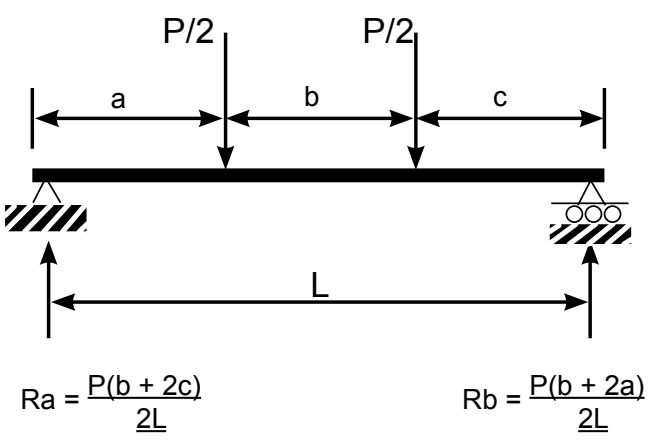

Figura 8. Modelo de los ensayos a flexión.

Fuente: Rusinque (2007). 
Tabla 7. Valores de módulos de elasticidad de diferentes investigaciones

\begin{tabular}{lcccc}
\hline $\begin{array}{c}\text { Módulo de elasticidad } \\
\text { (MPa) }\end{array}$ & NSR 10 & $\begin{array}{c}\text { Lozano, Luna y } \\
\text { Takeuchi (2010) }\end{array}$ & Ardila (2013) & $\begin{array}{c}\text { Resultado del } \\
\text { proyecto }\end{array}$ \\
\hline Promedio E0.5 & 9500 & 13904 & 14933 & 15483 \\
Percentil 5 E0.05 & 7500 & 7829 & 11586 & 12557 \\
Mínimo EMínimo & 4000 & 2996 & 11440 & 10526 \\
\hline
\end{tabular}

En la tabla 7 se observan valores obtenidos de módulo de elasticidad para la Guadua angustifolia ensayada a flexión. Los valores mayores fueron obtenidos en esta investigación. Para confirmar dicha superioridad, conviene mencionar que el contenido de humedad promedio registrado por Lozano et ál. (2010) fue de $71.04 \%$ y que el de Ardila (2013) fue de $19.91 \%$, resultados inferiores al $102.2 \%$ registrado en este estudio. Los esfuerzos admisibles encontrados por Lozano et ál. (2010), Ardila (2013) y Osorio y Sapuyes (2017) fueron de $12.46 \mathrm{MPa}, 10.19 \mathrm{MPa}$ y $13.85 \mathrm{MPa}$, respectivamente. Además, se debe tener en cuenta el método empleado para la determinación del módulo de elasticidad. Lozano et ál. (2010) y Ardila (2013) usaron la ecuación 5.

$$
E=\frac{23 * F^{*} L^{3}}{1296 * \delta^{*} I_{B}}
$$

Donde:

$\delta \quad$ : deflexión en el punto medio de la luz

$\mathrm{F}$ : carga máxima aplicada

L : luz libre

$\mathrm{I}_{\mathrm{B}}$ : momento de inercia

Prieto y Sánchez (2002) y Rusinque (2007) implementaron los procedimientos estipulados en la ISO 22157 (los resultados que se muestran en la tabla 8). Los autores utilizaron probetas con luces libres de $0.50 \mathrm{~m}, 1.0 \mathrm{~m}, 1.50 \mathrm{~m}, 2.0 \mathrm{~m}, 2.50 \mathrm{~m}$ y $3.0 \mathrm{~m}$. El primer estudio tuvo un esfuerzo máximo a flexión de 58.60 MPa, y el segundo de 109.55 MPa a luz libre de $3.0 \mathrm{~m}$. Se observa que estos resultados son diferentes a los obtenidos por el proyecto tomado como referencia en esta investigación.

Tabla 8. Módulos de elasticidad a luz libre de $3.0 \mathrm{~m}$

\begin{tabular}{lcc}
\hline \multicolumn{1}{c}{ Módulo de elasticidad (MPa) } & Prieto y Sánchez (2002) & Rusinque (2007) \\
\hline Promedio E0.5 & 15393 & \\
Percentil 5 E0.05 & 14916 & 15151 \\
Mínimo EMínimo & 9286 & \\
\hline
\end{tabular}

La tabla 9 muestra los resultados obtenidos en investigaciones realizadas en Brasil en dos especies diferentes de bambú.

Tabla 9. Resultados investigaciones en Brasil

\begin{tabular}{lccc}
\cline { 2 - 3 } & $\begin{array}{c}\text { Strzelecki, Klitzke, Batista, do } \\
\text { Nascimento y Ostapiv (2013) }\end{array}$ & \multicolumn{2}{c}{ Dos Reis (2012) } \\
\cline { 2 - 4 } Módulo de elasticidad (MPa) & 13719 & 15600 & con nodo \\
Resistencia última a flexión & 167 & 167 & 12300 \\
(MOR) (MPa) & Bambu-mossô (Phyllostachys pubescens) & Bambú gigante (Dendrocalamus giganteus) \\
Bambú &
\end{tabular}


Ordóñez-Candelaria y Bárcenas Pazos (2014) determinaron los valores de resistencia en flexión (MOR) y módulo de elasticidad en tres especies de guaduas mexicanas:

En condición verde, las resistencias fueron en general, más bajos para la parte basal y más altos en la parte superior. Para Guadua velutina fueron entre $75.5 \mathrm{MPa}$ y $88.3 \mathrm{MPa}$; para Guadua amplexifolia de 77.0 MPa y $102.1 \mathrm{MPa}$; y para Guadua aculeata de $59.7 \mathrm{MPa}$ a $89.2 \mathrm{MPa}$. (p. 111)

De igual manera, los módulos de elasticidad fueron en general más bajos para la parte basal y más altos en la parte superior, $15400 \mathrm{MPa}$ a $19600 \mathrm{MPa}, 14500 \mathrm{MPa}$ a $22700 \mathrm{MPa}$ y $13800 \mathrm{MPa}$ a $19500 \mathrm{MPa}$, respectivamente (Ordóñez-Candelaria y Bárcenas-Pazos, 2014)

Zaragoza-Hernández et ál. (2015) determinaron la resistencia última a flexión y su módulo de elasticidad en guadua mexicana (Guadua aculeata), obteniendo valores de 51.9 a $79.6 \mathrm{MPa}$ para la resistencia y 15100 a $24000 \mathrm{MPa}$ para el módulo de elasticidad.

\section{Conclusiones}

Comparando los resultados obtenidos de módulo de elasticidad promedio $\left(\mathrm{E}_{0.5}\right)$, percentil $5\left(\mathrm{E}_{0.05}\right)$ y mínimo $\left(\mathrm{E}_{\min }\right)$ en Guadua angustifolia proveniente del municipio de Pitalito $\left(\mathrm{E}_{0.5}=15483 \mathrm{MPa}\right.$, $\mathrm{E}_{0.05}=12557 \mathrm{MPa}$ y $\left.\mathrm{E}_{\min }=10526 \mathrm{MPa}\right)$, con lo estipulados en la NSR $10\left(\mathrm{E}_{0.5}=9500 \mathrm{MPa}\right.$, $\mathrm{E}_{0.05}=7500 \mathrm{MPa} \mathrm{y} \mathrm{E}_{\min }=4000 \mathrm{MPa}$ ), se observa una diferencia estadísticamente significativa, contrario a lo que pasa con los resultados de Lozano et ál. (2010) y Ardila (2013), en los que la diferencia es mínima, a pesar de que la procedencia de las probetas es distinta, así como las condiciones edafológicas y climatológicas que caracterizan a cada región.

Los contenidos de humedad para los estudios a flexión realizados en Colombia sobre Guadua angustofolia presentan diferencias significativas: $71.04 \%$ para Lozano et ál. (2010), $19.91 \%$ para Ardila (2013) y $102.2 \%$ para Sapuyes y Osorio (2017).

Los esfuerzos admisibles presentados por Sapuyes y Osorio (2017) son superiores a los de Lozano et ál. (2010) y Ardila (2013), por lo que se puede determinar que la Guadua angustifolia proveniente del departamento del Huila cuenta con una ventaja representativa respecto al material procedente de los departamentos de Cundinamarca, Quindío, Tolima y Valle del Cauca.

Debido al cambio de normatividad, se encontraron diferencias en los procedimientos aplicados en las distintas investigaciones; no obstante, se puede afirmar que Guadua angustifolia proveniente del departamento del Huila, específicamente, de los municipios de Pitalito y Timaná, presentó condiciones favorables en cuanto a resistencia última a flexión y módulo de elasticidad a flexión. Por esta razón se recomienda continuar con las investigaciones, promover la siembra y la conservación de la especie y realizar estudios relacionados con la comercialización para su uso como material para la construcción.

\section{Referencias}

Alarcón, J., y Olarte, J., (2013). Esfuerzo máximo de tensión paralela a la fibra y determinación del módulo de elasticidad de la Guadua angustifolia del municipio de Pitalito-Huila (proyecto de grado). Universidad Surcolombiana, Neiva, Colombia.

Ardila C. (2013). Determinación de los valores de esfuerzos admisibles del bambú Guadua angustifolia Kunth del departamento de Tolima, Colombia (tesis de maestría). Maestría en Construcción, Facultad de Artes, Universidad Nacional de Colombia, Bogotá, Colombia. 
Capera, A., y Erazo, W. (2012). Resistencia a la compresión paralela a la fibra y determinación del módulo de elasticidad de la Guadua angustifolia del municipio de Pitalito-Huila (proyecto de grado). Universidad Surcolombiana, Neiva, Colombia.

Chavarro, S. (2016). Deflexiones de la Guadua angustifolia Kunth para culmos sometidos a esfuerzos por flexión con cargas permanentes en el ambiente de Bogotá. Modelo de Burger y modelo de Findley (tesis de maestría). Maestría en Ingeniería, Facultad de Ingeniería, Universidad Nacional de Colombia, Bogotá, Colombia.

Córdoba, K. (2009). Resistencia natural de la Guadua angustifolia Kunth al ataque de hongos ligninoliticos como alternativa hacia nuevas posibilidades de uso. Bogotá: Pontificia Universidad Javeriana.

Dos Reis, M. (2012). Introdução de espécies, manejo, caracterização e aplicações. Bauruk (tesis). Facultad de Ingeniería, Universidad Estatal Paulista, São Paulo, Brasil.

Duarte, M., Takeuchi, C., y Erazo, W. (2013). Análisis comparativo en muestras de Guadua angustifolia Kunth solicitadas a compresión paralela a la fibra. Ingeniería y Región, 10, 117-124.

González, H., Montoya, J., y Bedoya, J. (2008). Comportamiento de muestras de Guadua angustifolia Kunth con diafragma y sin diafragma sometidas a esfuerzo de compresión. Scientia et Technica, 38, 449-454.

Instituto Colombiano de Normas Técnicas y Certificación (2007). NTC 5525. Métodos de Ensayo para Determinar las Propiedades Fisicas y Mecánicas de la Guadua angustifolia Kunth. Bogotá: ICONTEC.

Instituto Colombiano de Normas Técnicas y Certificación (2007). NTC 5301. Preservación y Secado del Culmo de Guadua angustifolia Kunth. Bogotá: ICONTEC.

Instituto Colombiano De Normas Técnicas y Certificación (2008). NTC 5300. Cosecha y Postcosecha del Culmo de Guadua angustifolia Kunth. Bogotá: ICONTEC

International Organization for Standardization. (2004). ISO 22157. Bamboo: determination of physical and mechanical properties on bamboo. Ginebra: ISO.

López, G., y Salcedo, J. (2016). Resistencia a compresión perpendicular y determinación del módulo de elasticidad circunferencial de la Guadua angustifolia Kunth del municipio de Pitalito (tesis). Universidad Surcolombiana, Neiva, Colombia.

Lozano, J., Luna, P., y Takeuchi, C. (2010). Validación de la Guadua angustifolia como material estructural para diseño, por el método de esfuerzos admisibles. Bogotá, Colombia: Universidad Nacional de Colombia

McClure, F. (1966). El bambú como material de construcción (1ª Ed.). Bogotá: [s. d.].

Ministerio de Ambiente, Vivienda y Desarrollo Territorial. (2010). NSR 10Reglamento Colombiano de Construcciones Sismo Resistente. Bogotá: Ministerio de Ambiente, Vivienda y Desarrollo Territorial.

Ministerio de Ambiente, Vivienda y Desarrollo Territorial, CVC, CORPOCALDAS, CARDER y CORTOLIMA. (2008). Norma Unificada para el Manejo y Aprovechamiento de la Guadua. Recuperado de http://www.bosquesflegt.gov.co/sites/default/files/publicaciones/norma\%20 guadua.pdf 
Ordóñez-Candelaria, V., y Bárcenas-Pazos, G. (2014). Propiedades físicas y mecánicas de tres especies de guaduas mexicanas (Guadua aculeata, Guadua amplexifolia y Guadua velutina). Madera y Bosques, 20(2), 111-125.

Osorio, J., y Sapuyes, E. (2017). Esfuerzo de flexión y módulo de elasticidad de la Guadua angustifolia Kunth procedente del municipio de Pitalito, Huila (proyecto de grado). Universidad Surcolombiana, Neiva, Colombia.

Prieto, E., y Sánchez, J. (2002). Comportamiento de la Guadua angustifolia sometida a flexión (proyecto de grado). Facultad de Ingeniería Civil, Universidad Nacional de Colombia, Bogotá, Colombia.

Reyes, E., y Rayo G. (2014). Comportamiento a flexión de vigas de madera de gran longitud de sección compuesta reforzadas con láminas pegadas prensadas de Guadua angustifolia Kunth (trabajo de grado). Facultad de Ingeniería Civil, Universidad Militar Nueva Granada, Bogotá, Colombia.

Brand, R., Lozano, N., y Ruíz, D. Ronald. (2015). Caracterización física y mecánica de la guadua rolliza de la especie angustifolia Kunth mediante procesamiento digital de imágenes (trabajo de grado). Facultad de Ingeniería Civil, Universidad Militar Nueva Granada, Bogotá, Colombia.

Rusinque, M. (2007). Influencia de la perforación, para inmunización en los tabiques, en el comportamiento de elementos solicitados a flexión de Guadua angustifolia (tesis). Universidad Nacional de Colombia, Bogotá, Colombia.

Statgraphics. (2014). Statgraphics Centurión XVI [software versión de prueba 16.1.18]. Recuperado de http://www.statgraphics.com/

Strzelecki. R., Klitzke, R., Batista, D., do Nascimento, E., y Ostapiv, F. (2013). Resistência à flexão estática e à compressão paralela do bambu-mossô (Phyllostachys pubescens). Floresta, 43(3), 485-494.

Vélez, G. (2001). El Humilde bambú “acero vegetal”. En Memorias Congreso Virtual de Arquitectura. Presentado en Caracas, Venezuela.

Villegas, F. (2005). Comparación consumo de recursos energéticos en la construcción de vivienda social: guadua vs. concreto (tesis de maestría). Universidad Nacional de Colombia, Manizales, Colombia.

Zaragoza-Hernández, I., Ordóñez-Candelaria, V., Bárcenas-Pazos, G., Borja-de la Rosa, A., y Zamudio-Sánchez, F. (2015). Propiedades físico-mecánicas de una guadua mexicana (Guadua aculeata). Maderas. Ciencia y Tecnologia, 17(3), 505-516. 
